\title{
The retinal vasculature pathophysiological changes in vision recovery after treatment for indirect traumatic optic neuropathy patients
}

\author{
Yang Gao ${ }^{1} \cdot$ Jinmiao $\mathrm{Li}^{1} \cdot$ Huan $\mathrm{Ma}^{1} \cdot$ Cong Nie ${ }^{1} \cdot \mathrm{Xi} \mathrm{Lv}^{1} \cdot{\text { Xiaofeng } \mathrm{Lin}^{1} \cdot \text { Guangwei Luo }}^{1} \cdot$ Jianbo Shi ${ }^{2} \cdot \mathrm{Rong} \mathrm{Lu}^{1}$ (D)
}

Received: 6 January 2021 / Revised: 31 March 2021 / Accepted: 19 April 2021 / Published online: 11 May 2021

(c) The Author(s) 2021

\begin{abstract}
Purpose To evaluate the retinal vasculature pathophysiological changes of indirect traumatic optic neuropathy (ITON) patients after effective surgery.

Methods Monocular ITON patients who underwent endoscopic trans-ethmosphenoid optic canal decompression (ETOCD) or conservative treatments in Zhongshan Ophthalmic Center from January 2017 to June 2020 were recruited. Visual acuity (VA), visual evoked potential (VEP), oxygen saturation of retinal blood vessels $\left(\mathrm{SO}_{2}\right)$, and optical coherence tomography angiography (OCT-A) were measured. All patients were followed up at least 3 months after treatments.

Results A total of 95 ITON patients were recruited, including 77 patients who underwent ETOCD and 18 patients who underwent conservative treatments. After treatments, more patients received ETOCD $(59 / 77=76.6 \%)$ presented with improved VA compared with the patients with conservative treatments $(6 / 18=33.3 \%)$. Compared with the pre-therapeutic measurements, VEP were significantly improved after surgery in ETOCD-treated patients $(P<0.05)$. Latent periods of P1 and N2, as well as amplitude of P2 of VEP parameters, showed more sensitive to vision recovery $(P<0.05)$. Retinal artery $\mathrm{SO}_{2}$ and the differences between arteries and veins were improved in ETOCD-treated patients $(P<0.05)$. Meanwhile, with OCT-A examination, the retinal thickness and retinal vessel density were notably better in ETOCD-treated patients after surgery than that in patients received conservative treatments $(P<0.05)$.

Conclusions Vision recovery after effective treatment of ITON patients was associated with the increased oxygen saturation of retinal vessels, better availability of oxygen in the retina, greater vessel density, and thicker retinas, which might further underlie the vasculature mechanism of vision recovery in ITON patients.
\end{abstract}

\section{Key messages}

- The retinal vasculature pathophysiological changes in vision recovery after treatment for indirect traumatic optic neuropathy (ITON) patients has not yet been thoroughly studied.

- Vision recovery after effective treatment of ITON patients was associated with the increased oxygen saturation of retinal vessels and better availability of oxygen in the retina, which might further underly the vasculature mechanism of vision recovery in ITON patients.

- The retinal thickness and retinal vessel density were significantly greater in surgical patients after surgery than that in patients received conservative treatments, suggesting that the effective treatment of ITON mitigates the progress of retinal atrophy of severe ITON.

Keywords Vision recovery · Vasculature $\cdot$ Indirect traumatic optic neuropathy $\cdot$ Retinal oxygen saturation · Optical coherence tomography angiography

Rong Lu

lurong@gzzoc.com; rongluzz@yahoo.com

Extended author information available on the last page of the article 


\section{Introduction}

Indirect traumatic optic neuropathy (ITON) is an indirect acute injury of the optic nerve from nonpenetrating effects of craniofacial trauma, which occurs $2-5 \%$ of facial trauma and $0.5-2 \%$ of head trauma [1-3]. ITON is diagnosed for disruption of visual function with clinical characteristics that include a severe acute decrease of visual acuity, impaired visual evoked potential (VEP), and a relative afferent pupil defect.

The shock wave from concussive forces applied to the head or face is transmitted to the optic canal. It was hypothesized that swelling of the optic nerve within the fixed and limited cavity of the optic canal compromises blood supply, which exacerbates tissue ischemia and causes further damage to the injured optic nerve [4-6]. This pathophysiological hypothesis is commonly cited as a rationale for performing optic canal decompressive surgery. Endoscopic trans-ethmosphenoid optic canal decompression (ETOCD), an effective and less invasive approach of optic canal decompression, has become a trend in recent decades. Various studies reported positive outcomes from ETOCD surgery for ITON cases [7-12]. However, the underlying principle of ETOCD has not yet been proven. The pathophysiological changes after ETOCD, especially the blood supply alteration to the optic nerve and retina, might reveal the underlying mechanism of the effective therapy for ITON.

The mechanism underlying ITON appears to be multifactorial, and vascular insufficiency might be an important factor as in other optic neuropathies. Noninvasive spectrophotometric retinal oximetry has been used to measure oxygen saturation in retinal arteries and veins [13]. Besides, optical coherence tomography angiography (OCT-A) is a noninvasive technique capable of qualitatively and quantitatively evaluating the retinal and choroidal microvascular perfusion. They are both noninvasive diagnostic tools and thereby interesting in evaluating hemodynamic changes in different eye conditions, such as glaucoma, diabetic retinopathy, age-related macular degeneration, and several optic neuropathies [14-23].

In this study, with the retinal oximetry and OCT-A examination, it is feasible to investigate the retinal structural and hemodynamic changes after the effective therapy, thereby revealing the retinal vasculature pathophysiological changes in the process of vision recovery for ITON patients.

\section{Methods}

\section{Ethics approval}

Ethical approval and patient consent were obtained before surgery and the procedures adhered to the tenets of the
1964 Declaration of Helsinki. Protocols were approved by the Institutional Ethics Committee (2019KYPJ155, Medical Ethics Committee, Zhongshan Ophthalmic Center, Guangzhou, Guangdong, China).

\section{Subjects}

This study recruited 95 patients with indirect traumatic optic neuropathy (ITON) in Zhongshan Ophthalmic Center from January 2017 to June 2020, including 77 patients underwent endoscopic trans-ethmosphenoid optic canal decompression (ETOCD) surgery and 18 patients underwent conservative treatments. The inclusion criteria were as follows: (1) patients were diagnosed with ITON; (2) patients' initial VA $\leq 0.3$; (3) patients underwent ETOCD surgery or conservative treatments within 3 months after the injury; (4) patients were followed up for at least 3 months; and (5) patients have no history of other severe ocular disorders or ocular surgery. The written informed consent was acquired from the patients. Details on gender, age, side, lag time from injury to surgery, causes of injuries, blood pressure, intraocular pressure, and axial length were recorded.

The diagnosis of ITON was made by traumatic history and examination, including (1) a closed head injury with no direct trauma to the optic nerve; (2) acute decrease of visual acuity; (3) a positive relative afferent pupil defect (RAPD); and (4) an abnormal visual evoked potential (VEP) with normal fundus examination.

\section{Treatments}

A total 95 patients were divided into the ETOCD group (77 patients) and the conservative group (18 patients) randomly. Doctors have had comprehensively informed the patients about the possible outcomes/risks of treatment options. Treatment for ITON was performed with the patient's consent.

The indications for ETOCD were as follows: (1) patients were diagnosed with ITON; (2) patients' initial VA $\leq 0.3$; (3) the lag time from the injury to the surgery was less than 3 months; (4) patients have no history of other severe systemic disorders; and (5) the written informed consent was acquired from the patients.

All ETOCD surgeries were performed under general anesthesia. The surgery was performed in the following four steps. (1) Expose the optic canal. (2) Open the optic canal. (3) Incise the annulus of Zinn. (4) Cover the optic nerve with nerve growth factor.

The conservative treatments included observation and neurotrophic drugs. 


\section{Ophthalmic and systemic examination}

All subjects underwent comprehensive ocular examinations, including best-corrected visual acuity (BCVA), axial length measurement, non-contact tonometer (NCT), slitlamp biomicroscopy, fundoscopy, apparent pupillary defect evaluation, and ocular motility assessment. Blood pressure was measured after at least 5 min of resting. Mean arterial blood pressure was computed as diastolic blood pressure plus one-third of pulse pressure.

To evaluate the vision changes after treatments, visual acuity (VA) was measured before and after treatments at various timepoints, i.e., 1 day, 2 days, 3 days, 1 week, 2 weeks, 1 month, and 3 months, respectively. The therapeutic effectiveness was defined by an increase of VA, wider visual fields, and/or brighter visual fields.

\section{Visual evoked potential (VEP)}

Flash VEP was measured before treatments and followed up for 3 months after treatments (RETI-Port, Roland Consult, Brandenburg, Germany).

\section{Oxygen saturation of retinal blood vessels $\left(\mathrm{SO}_{2}\right)$}

$\mathrm{SO}_{2}$ using Oxymap $\mathrm{T} 1$ retinal oximeter (Oxymap ehf., Reykjavik, Iceland) was measured before and after treatments in 1 day, 1 week, 2 weeks, 1 month, and 3 months, respectively.

Oxymap T1 is a noninvasive instrument used for measuring in vivo oxygen saturation of retinal arteries $\left(\mathrm{SaO}_{2}\right)$ and veins $\left(\mathrm{SvO}_{2}\right)$, as well as the differences between the arteries and veins ( $\mathrm{AVD}, \mathrm{SaO}_{2}-\mathrm{SvO}_{2}$ ). The theoretical basis of Oxymap T1 is the difference in light absorbance of deoxyhemoglobin and oxyhemoglobin at specific wavelengths (570 and $600 \mathrm{~nm}) . \mathrm{SO}_{2}$ values were calculated using a computerized algorithm after collecting fundus images at these two wavelengths. After the participants reached complete cycloplegia, Oxymap examination was conducted following standard procedures (version from November 21, 2013).

\section{Optical coherence tomography angiography (OCT-A)}

All subjects were examined under a single OCT-A system (AngioVue; Optovue, Inc., Fremont, CA, USA), which was able to visualize and quantify the microvasculature in the retina. The scanning speed of the device was 70,000 A-scans per second, and the central wavelength was $840 \mathrm{~nm}$. Each studied eye underwent a $3.0 \times 3.0 \mathrm{~mm}$ cube angio scan centered on the optic nerve head and a $3.0 \times 3.0 \mathrm{~mm}$ macular cube angio scan centered on the fovea. A split spectrum amplitude decorrelation angiography algorithm was applied to produce OCT-A images.

Vessel density was defined as the percentage of perfused vascular area in relation to the whole selected region in en face views. All vessel density parameters were automatically calculated using the built-in program. Peripapillary vessel density parameters were obtained from the $3.0 \times 3.0 \mathrm{~mm}$ cube angio scan centered on the optic nerve head. The macular whole image vessel density was generated from the $3.0 \times 3.0 \mathrm{~mm}$ cube angio scan centered on the fovea. The optic nerve head scanning area was further divided into the inside disc and peripapillary fields. The macular scanning area was segmented into three areas: fovea, parafoveal, and perifoveal zone. The parafoveal and perifoveal zone was further divided into 4 sessions by an annular grid: temporal, superior, nasal, and inferior.

Apart from vessel density indices, thickness of the retinal nerve fiber layer (RNFL) of optic nerve head area was measured automatedly. The optic nerve head scanning area was divided into 4 sessions by an annular grid: temporal, superior, nasal, and inferior.

\section{Statistical analysis}

Examination operators for VEP, $\mathrm{SO}_{2}$, and OCT-A as well as the statistician were blinded from patients' details and treatment groups.

Continuous variables were described as means \pm standard deviation (SD). A paired $t$ test was performed to compare patients' VA, VEP measures, retinal oxygen saturation, and OCT-A measurements before and after treatments. A $t$ test was performed to compare the parameters between different groups, and a Bonferroni correction was applied if necessary. Statistical analysis was performed with statistical software (SPSS version 20; IBM Corp., Armonk, NY, USA). Statistical significance was defined with $P$ value of less than 0.05 .

\section{Results}

\section{Baseline characteristics of patients}

A total of 95 patients with monocular indirect traumatic optic neuropathy (ITON), including 77 patients treated with endoscopic trans-ethmosphenoid optic canal decompression (ETOCD) surgery and 18 patients who underwent conservative treatments, were recruited in the study. Participant characteristics are listed in Table 1. In 77 ETOCD-treated patients, 68 were male $(88.3 \%)$ and 9 were female $(11.7 \%)$. The mean age of all patients was $26.9 \pm 14.1$ years old (range 5-62). The affected eyes were 38 right eyes and 39 left eyes. To avoid the patients' selection bias, the initial VA, lag time from the injury to the therapy, sex, age, and causes of injuries of these two treatment groups were analyzed statistically using $t$ test. And these parameters between these two groups were matched (all $P>0.05$; Table 1). 
Table 1 Clinical characteristics of 95 enrolled subjects and included eyes

\begin{tabular}{lll}
\hline Variables & ETOCD & Conservative treatment \\
\hline Patients & 77 & 18 \\
Sex, male & $68(88.3 \%)$ & $16(88.9 \%)$ \\
Age (years) & $26.9 \pm 14.1(5-62)$ & $29.0 \pm 17.8(10-59)$ \\
Side, right & $38(49.4 \%)$ & $9(50.0 \%)$ \\
Lag time (days) & $20.0 \pm 16.9(3-80)$ & $23.2 \pm 16.0(3-62)$ \\
Causes of injuries & & \\
$\quad$ Traffic accidents & $46(59.7 \%)$ & $10(55.6 \%)$ \\
$\quad$ Falls & $21(27.3 \%)$ & $6(33.3 \%)$ \\
$\quad$ Fights/assaults & $10(13.0 \%)$ & $2(11.1 \%)$ \\
Initial VA & & \\
$\quad$ VA, range & NLP to 0.3 & NLP to 0.3 \\
$\quad$ logMAR VA & $-3.38 \pm 1.42$ & $-3.45 \pm 1.67$ \\
SBP (mmHg) & $121.7 \pm 9.2(92-156)$ & $122.6 \pm 10.7(94-156)$ \\
DBP (mmHg) & $75.5 \pm 7.2(56-95)$ & $75.1 \pm 6.3(56-92)$ \\
MABP (mmHg) & $90.9 \pm 7.3(74-125)$ & $90.6 \pm 7.1(74-123)$ \\
IOP (mmHg) & $14.6 \pm 3.7(7.7-32)$ & $14.3 \pm 5.1(8-32)$ \\
Axial length (mm) & $23.6 \pm 0.7(21.3-26.4)$ & $23.5 \pm 0.7(21.3-26.4)$ \\
\hline
\end{tabular}

ETOCD, endoscopic trans-ethmosphenoid optic canal decompression; lag time, lag time from the injury to the treatment; $V A$, visual acuity; $S B P$, systolic blood pressure; $D B P$, diastolic blood pressure; $M A B P$, mean arterial blood pressure; $I O P$, intraocular pressure

\section{Vision was efficiently improved in ITON patients after surgery}

After ETOCD, 76.6\% (59/77) patients achieved VA improvement. Meanwhile, the percentage of patients with VA improvement after the conservative treatment was 33.3\% (6/18). The effectiveness was defined by increased VA, wider visual fields, or even brighter visual fields.

The proportions of VA improvement in total 77 ITON patients were timely increasing at 58.4, 66.2, 67.5, 70.1, 75.3 , and $76.6 \%$ in 1 day (D1), 2 days (D2), 3 days (D3), 1 week (W1), 1 month (M1), and 3 months (M3), respectively. This clinical observation shows the rapid and steady effectiveness of ETOCD in ITON patients.

There was no difference between the initial logMAR VA before treatments of two groups (ETOCD group, $-3.45 \pm 1.42$; conservative treatment group, $-3.38 \pm 1.67 ; P>0.05)$. Nevertheless, after 3 months of two different therapies, $\log$ MAR VA was significant between ETOCD group and conservative treatment group $(-2.48 \pm 1.61$ vs $-3.13 \pm 1.72, P<0.05)$.

\section{The visual evoked potential (VEP) was significantly improved in ITON patients treated with ETOCD surgery, especially in patients with vision recovery after surgery}

The latent periods of N1, N2, and P1 for VEP were significantly decreased, and the amplitudes of $\mathrm{P} 1$ and $\mathrm{P} 2$ were significantly increased after ETOCD surgery in all ITON patients (all $P<0.001$, Table 2), and these above parameters after treatments of ETOCD-treated patients were significantly better than those of conservative therapeutic patients, suggesting VEP was significantly improved after ETOCD.

Moreover, to explore if VEP change was related to surgical effectiveness, based on surgery effectiveness, ITON patients were divided into two groups: with $(n=59)$ or without $(n=18)$ VA improvement after ETOCD (Table 2). All VEP parameters, including the latent period of N1, $\mathrm{N} 2$, P1, and P2, as well as the amplitude of P1 and P2, were not statistically different between these two groups before ETOCD. Notably, in patients with VA improvement, the latent period of $\mathrm{P} 1$, the latent period of $\mathrm{N} 2$, and the amplitude of $\mathrm{P} 2$ were improved significantly (decreased from $89.01 \pm 16.90$ to $65.63 \pm 18.74 \mathrm{~ms}$, decreased from $111.57 \pm 20.37$ to $82.01 \pm 18.35 \mathrm{~ms}$, and increased from $4.60 \pm 1.91$ to $6.92 \pm 3.02 \mu \mathrm{V}$, respectively, all $P<0.001$ ), and they were not significantly improved in patients without VA improvement. The results discovered that above three VEP parameters were critical and significantly related to VA improvement after ETOCD. Nevertheless, VEP results were not of correlation with age, gender, side of injury, and courses of injuries $(P>0.05)$.

\section{The retinal oxygen saturation was significantly improved in ITON patients treated with ETOCD surgery, especially in patients with vision recovery after surgery}

The oxygen saturation of retinal arteries $\left(\mathrm{SaO}_{2}\right)$ and veins $\left(\mathrm{SvO}_{2}\right)$ and the differences between the arteries and veins (AVD, $\mathrm{SaO}_{2}-\mathrm{SvO}_{2}$ ) were measured as the main outcomes. For the patients underwent ETOCD surgery, compared to the pre-operative $\mathrm{SaO}_{2}(86.91 \pm 6.63 \%)$, the $\mathrm{SaO}_{2}$ increased significantly throughout D1 $(88.97 \pm 6.25 \%$, $P=0.002)$ to $\mathrm{M} 3(93.94 \pm 4.69 \%, P<0.001)$ in all ITON patients after ETOCD surgery (Fig. 1a). Similarly, the post-operative $\mathrm{SvO}_{2}$ increased significantly throughout $\mathrm{W} 1(57.17 \pm 5.36 \%, P<0.001)$ to $\mathrm{M} 3(57.82 \pm 4.67 \%$, $P<0.001)$, compared with pre-operative $\mathrm{SvO}_{2}$ $(53.79 \pm 7.00 \%)$ (Fig. 1b). The pre-operative AVD was $33.07 \pm 5.08 \%$, and the post-operative AVD increased significantly throughout $\mathrm{D} 1(33.91 \pm 7.16 \%, P=0.004)$ to M3 $(37.91 \pm 4.57 \%, P<0.001)$ (Fig. 1c).

However, for the patients treated with conservative therapy, there was no difference between these above parameters of pre-treatment and post-treatment (Fig. 1). Besides, $\mathrm{SaO}_{2}$ (Fig. 1a) and AVD (Fig. 1c) of ETOCD-treated patients 3 months after the surgery were significantly improved than that of conservative therapeutic patients $(P=0.003$ and $P<0.001$, respectively). 
Table 2 The flash visual evoked potential (VEP) of indirect traumatic optic neuropathy (ITON) patients before and 3 months after endoscopic trans-ethmosphenoid optic canal decompression (ETOCD) and the conservative treatment

\begin{tabular}{|c|c|c|c|c|}
\hline \multirow[t]{2}{*}{ Variables } & \multirow[t]{2}{*}{ Groups } & \multicolumn{3}{|l|}{ Time points } \\
\hline & & Pre-treatment & Post-treatment & $P$ \\
\hline \multirow[t]{6}{*}{ Latent period of N1 (ms) } & ETOCD-Improved VA & $69.60 \pm 14.44$ & $50.21 \pm 20.69$ & $<0.001 *$ \\
\hline & ETOCD-No improved VA & $76.00 \pm 19.44$ & $54.01 \pm 18.51$ & $<0.001^{*}$ \\
\hline & $P^{\dagger}$ & 0.085 & 0.308 & \\
\hline & ETOCD-Total & $71.13 \pm 18.92$ & $51.12 \pm 16.74$ & $<0.001 *$ \\
\hline & Conservative treatment & $69.70 \pm 19.17$ & $66.24 \pm 17.58$ & 0.211 \\
\hline & $P^{\#}$ & 0.756 & $0.001 *$ & \\
\hline \multirow[t]{6}{*}{ Latent period of N2 (ms) } & ETOCD-Improved VA & $111.57 \pm 20.37$ & $82.01 \pm 18.35$ & $<0.001 *$ \\
\hline & ETOCD-No improved VA & $114.51 \pm 11.25$ & $107.43 \pm 10.62$ & 0.428 \\
\hline & $P^{\dagger}$ & 0.245 & $<0.001^{*}$ & \\
\hline & ETOCD-Total & $111.80 \pm 19.69$ & $87.51 \pm 14.13$ & $<0.001^{*}$ \\
\hline & Conservative treatment & $109.25 \pm 15.98$ & $100.48 \pm 24.59$ & 0.050 \\
\hline & $P^{\#}$ & 0.714 & $<0.001^{*}$ & \\
\hline \multirow[t]{6}{*}{ Latent period of P1 (ms) } & ETOCD-Improved VA & $89.01 \pm 16.90$ & $65.63 \pm 18.74$ & $<0.001^{*}$ \\
\hline & ETOCD-No improved VA & $92.11 \pm 11.09$ & $90.94 \pm 22.43$ & 0.347 \\
\hline & $P^{\dagger}$ & 0.301 & $0.009 *$ & \\
\hline & ETOCD-Total & $89.50 \pm 16.77$ & $72.30 \pm 27.53$ & $<0.001^{*}$ \\
\hline & Conservative treatment & $88.47 \pm 15.36$ & $85.57 \pm 25.36$ & 0.397 \\
\hline & $P^{\#}$ & 0.847 & $0.013^{*}$ & \\
\hline \multirow[t]{6}{*}{ Latent period of P2 (ms) } & ETOCD-Improved VA & $128.31 \pm 25.51$ & $128.42 \pm 26.02$ & 0.987 \\
\hline & ETOCD-No improved VA & $129.77 \pm 15.19$ & $130.93 \pm 23.98$ & 0.765 \\
\hline & $P^{\dagger}$ & 0.715 & 0.662 & \\
\hline & ETOCD-Total & $128.65 \pm 20.81$ & $129.02 \pm 24.82$ & 0.207 \\
\hline & Conservative treatment & $125.69 \pm 22.58$ & $123.89 \pm 26.71$ & 0.514 \\
\hline & $P^{\#}$ & 0.152 & 0.101 & \\
\hline \multirow[t]{6}{*}{ Amplitude of P1 $(\mu \mathrm{V})$} & ETOCD-Improved VA & $3.42 \pm 1.41$ & $6.38 \pm 3.58$ & $<0.001 *$ \\
\hline & ETOCD-No improved VA & $4.48 \pm 2.31$ & $7.20 \pm 4.09$ & $0.001 *$ \\
\hline & $P^{\dagger}$ & 0.137 & 0.108 & \\
\hline & ETOCD-Total & $3.67 \pm 2.71$ & $6.58 \pm 4.16$ & $<0.001 *$ \\
\hline & Conservative treatment & $4.01 \pm 2.16$ & $4.28 \pm 3.12$ & 0.769 \\
\hline & $P^{\#}$ & 0.514 & $<0.001^{*}$ & \\
\hline \multirow[t]{6}{*}{ Amplitude of P2 $(\mu \mathrm{V})$} & ETOCD-Improved VA & $4.60 \pm 1.91$ & $6.92 \pm 3.02$ & $<0.001 *$ \\
\hline & ETOCD-No improved VA & $4.92 \pm 1.37$ & $4.43 \pm 2.83$ & 0.291 \\
\hline & $P^{\dagger}$ & 0.196 & $0.006^{*}$ & \\
\hline & ETOCD-Total & $4.69 \pm 1.46$ & $6.07 \pm 3.21$ & $<0.001 *$ \\
\hline & Conservative treatment & $4.93 \pm 2.47$ & $4.81 \pm 4.19$ & 0.844 \\
\hline & $P^{\#}$ & 0.845 & $0.041 *$ & \\
\hline
\end{tabular}

Data are the means plus/minus standard deviations. ETOCD, endoscopic trans-ethmosphenoid optic canal decompression; $V A$, visual acuity; $P, P$ value between values of pre-treatment and post-treatment; $P^{\dagger}, P$ value between improved VA group and no improved VA group after ETOCD; $P^{\#}, P$ value between two groups treated with ETOCD and the conservation treatment; $* P<0.05$
Meanwhile, the diameter and length of arteries and veins did not show any difference between pre-therapeutic and post-therapeutic measurements in both treatment groups $(P>0.05$, Table 3$)$.

Moreover, to explore if the change of oxygen saturation of retinal vessels was related to VA improvement, we divided all ITON patients into two groups based on vision recovery after surgery (Fig. 2). While compared between the two groups, $\mathrm{SaO}_{2}$ was found significantly increased with VA-improved group, from $\mathrm{W} 1(93.30 \pm 6.24$ vs $88.13 \pm 3.90, P=0.03)$ to $\mathrm{M} 3$ $(95.04 \pm 2.94$ vs $91.76 \pm 3.62, P<0.01$, Fig. $2 b$,). Notably, AVD, which may imply the use of oxygen by cells, was also significantly increased in patients with VA improvement than those without, throughout W1 $(38.80 \pm 6.90$ vs $31.53 \pm 6.88, P=0.02)$ to 3 months $(38.45 \pm 4.96$ vs 
a

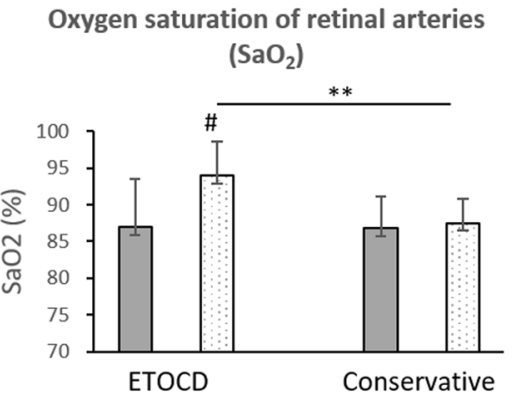

b

Oxygen saturation of retinal veins $\left(\mathrm{SvO}_{2}\right)$

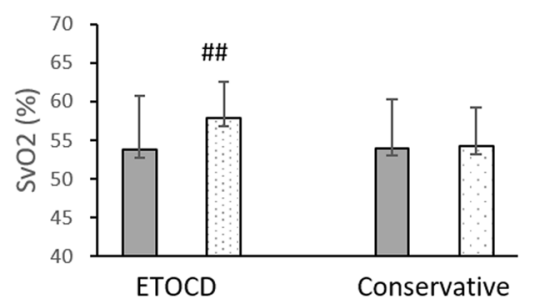

c

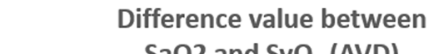

$\mathrm{SaO} 2$ and $\mathrm{SvO}_{2}$ (AVD)

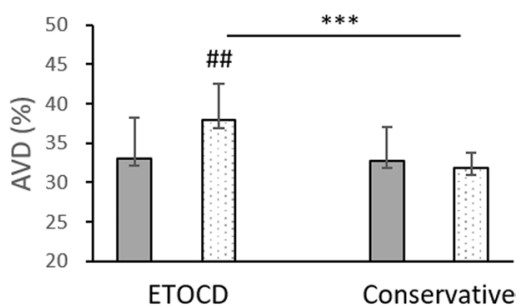

Pre-treatment $\square \mid$ Three Months Post-treatment

Fig. 1 Oxygen saturation of retinal blood vessels of indirect traumatic optic neuropathy (ITON) patients before and 3 months after endoscopic trans-ethmosphenoid optic canal decompression (ETOCD) and the conservative treatment. a $\mathrm{SaO}_{2}$, b $\mathrm{SvO}_{2}$, and c AVD before and after treatments in ETOCD-treated patients and conservative thera- peutic patients, respectively. ${ }^{\#} P<0.05 ;{ }^{\# \#} P<0.01$ between values of pre-treatment and 3 months post-treatment. $* * P<0.01$; $* * * P<0.001$ between values of post-ETOCD and post-conservative therapy. $\mathrm{SaO}_{2}$, oxygen saturation of retinal arteries; $\mathrm{SvO}_{2}$, oxygen saturation of retinal veins; $A V D$, the difference value between $\mathrm{SaO}_{2}$ and $\mathrm{SvO}_{2}$
Table 3 Diameter and length of retinal blood vessels in indirect traumatic optic neuropathy (ITON) patients before and 3 months after endoscopic trans-ethmosphenoid optic canal decompression (ETOCD) and the conservative treatment

\begin{tabular}{|c|c|c|c|c|}
\hline \multirow[t]{2}{*}{ Variables } & \multirow[t]{2}{*}{ Groups } & \multicolumn{3}{|l|}{ Time points } \\
\hline & & Pre-treatment & Post-treatment & $P$ \\
\hline \multirow[t]{6}{*}{ Diameter of arteries } & ETOCD-Improved VA & $11.86 \pm 1.29$ & $11.01 \pm 1.45$ & 0.054 \\
\hline & ETOCD-No improved VA & $12.44 \pm 2.68$ & $10.75 \pm 1.25$ & 0.112 \\
\hline & $P^{\dagger}$ & 0.245 & 0.293 & \\
\hline & ETOCD-Total & $12.00 \pm 1.45$ & $10.95 \pm 2.40$ & 0.100 \\
\hline & Conservative treatment & $12.04 \pm 2.14$ & $10.89 \pm 2.59$ & 0.100 \\
\hline & $P^{\#}$ & 0.847 & 0.798 & \\
\hline \multirow[t]{6}{*}{ Diameter of veins } & ETOCD-Improved VA & $16.12 \pm 2.31$ & $15.54 \pm 2.05$ & 0.072 \\
\hline & ETOCD-No improved VA & $15.98 \pm 2.31$ & $13.36 \pm 1.78$ & 0.212 \\
\hline & $P^{\dagger}$ & 0.729 & 0.086 & \\
\hline & ETOCD-Total & $16.09 \pm 2.30$ & $15.02 \pm 2.70$ & 0.112 \\
\hline & Conservative treatment & $16.89 \pm 2.59$ & $15.48 \pm 1.79$ & 0.278 \\
\hline & $P^{\#}$ & 0.347 & 0.654 & \\
\hline \multirow[t]{6}{*}{ Length of arteries } & ETOCD-Improved VA & $2373.89 \pm 1153.14$ & $2705.67 \pm 658.829$ & 0.031 \\
\hline & ETOCD-No improved VA & $2599.75 \pm 1422.22$ & $2262.50 \pm 859.50$ & 0.050 \\
\hline & $P^{\dagger}$ & 0.591 & 0.472 & \\
\hline & ETOCD-Total & $2428.10 \pm 1248.29$ & $2599.31 \pm 801.26$ & 0.102 \\
\hline & Conservative treatment & $2523.14 \pm 1312.25$ & $2565.66 \pm 924.02$ & 0.854 \\
\hline & $P^{\#}$ & 0.512 & 0.821 & \\
\hline \multirow[t]{6}{*}{ Length of veins } & ETOCD-Improved VA & $2562.22 \pm 730.41$ & $2810.33 \pm 733.80$ & 0.334 \\
\hline & ETOCD-No improved VA & $2398.00 \pm 736.23$ & $2607.00 \pm 556.34$ & 0.219 \\
\hline & $P^{\dagger}$ & 0.051 & 0.776 & \\
\hline & ETOCD-Total & $2522.807 \pm 756.54$ & $2761.53 \pm 568.80$ & 0.147 \\
\hline & Conservative treatment & $2489.26 \pm 893.36$ & $2619.26 \pm 783.25$ & 0.358 \\
\hline & $P^{\#}$ & 0.379 & 0.569 & \\
\hline
\end{tabular}

Data are the means plus/minus standard deviations. ETOCD, endoscopic trans-ethmosphenoid optic canal decompression; $V A$, visual acuity; $P, P$ value between values of pre-treatment and post-treatment; $P^{\dagger}, P$ value between improved VA group and no improved VA group after ETOCD; $P^{\#}, P$ value between two groups treated with ETOCD and the conservation treatment 

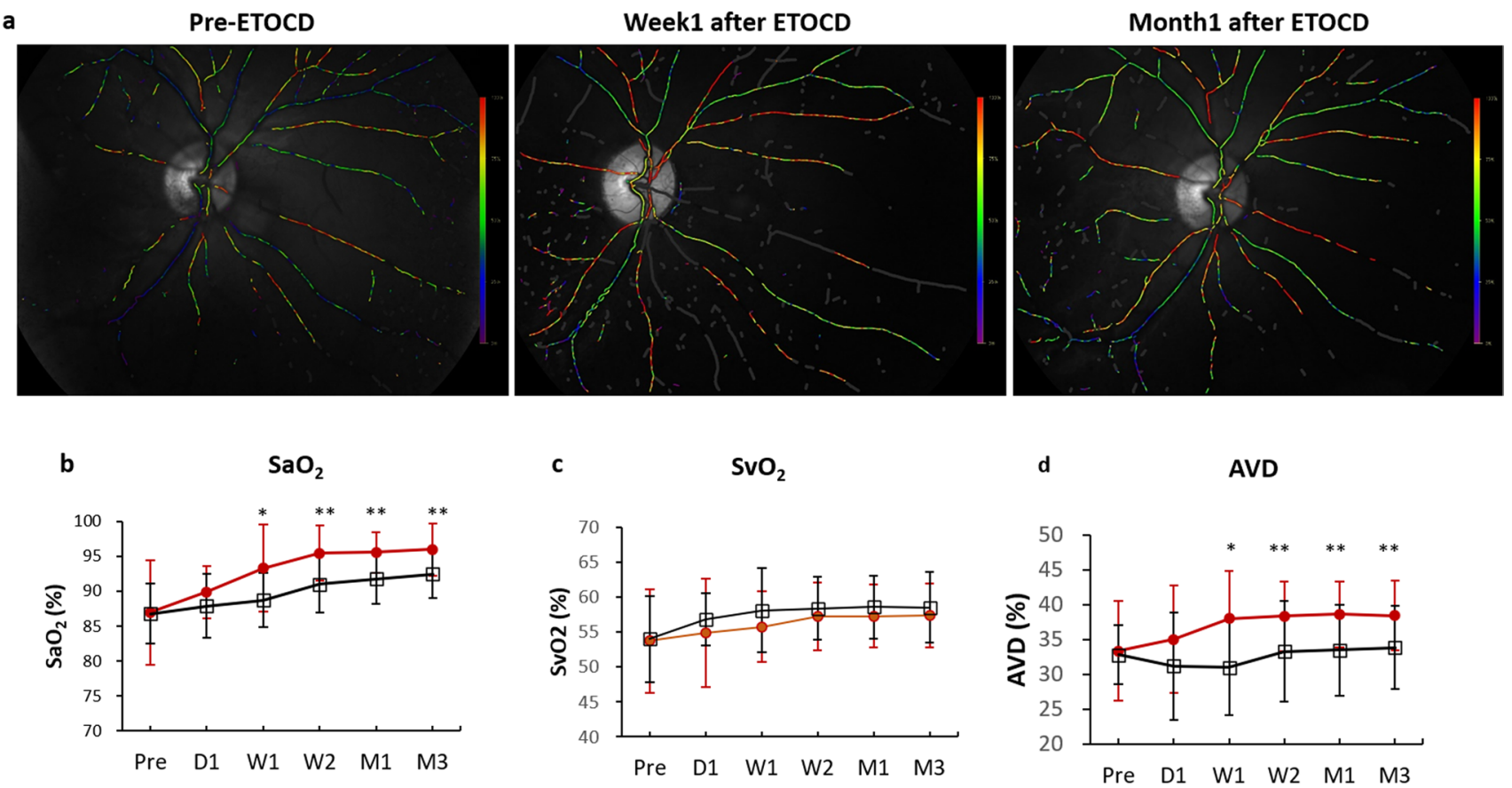

- VA improved after ETOCD

Fig. 2 The oxygen saturation of retinal vessels of indirect traumatic optic neuropathy (ITON) patients was improved after endoscopic trans-ethmosphenoid optic canal decompression (ETOCD). a The typical retinal oxygen saturation images of ITON patients before ETOCD (left), 1 week (middle) and 1 month after ETOCD (right). The redder the color, the higher the oxygen saturation of retinal vessels. Throughout follow-up timepoints from $\mathrm{D} 1$ to $\mathrm{M} 3, \mathbf{b} \mathrm{SaO}_{2}$ was

$27.87 \pm 1.96, P<0.01$, Fig. 2 d). However, for other parameters after ETOCD, including $\mathrm{SvO}_{2}$ as well as the diameter and length of arteries and veins, there was no significant difference between patients with or without post-operative VA improvement (Fig. 2c).

Meanwhile, the diameter and length of arteries and veins did not show any difference between ETOCD-treated patients with and without VA improvement after the surgery $(P>0.05$, Table 3$)$. These measures of the retinal oxygen saturation did not show any correlation with age, gender, side of injury, and causes of injuries $(P>0.05)$.

\section{VA improvement is associated with thicker retinas and greater vessel density in ITON patients after the surgery}

The retina thickness and the vessel density of optic nerve head area and macular before and 3 months after treatments were measured as the main outcomes using the optical coherence tomography angiography (OCT-A; Table 4, Figs. 3 and 4).
—VA not improved after ETOCD

significantly higher in patients with VA improvement than in those without VA improvements, $* P<0.05$; $* * P<0.01$; c $\mathrm{SvO}_{2}$ was not significantly different before and after ETOCD; and d AVD was significantly higher in patients with VA improvement than in those without VA improvements, $* P<0.05 ; * * P<0.01$. Post-surgery timepoints: after 1 day (D1), 1 week (W1), 2 weeks (W2), 1 month (M1), and 3 months (M3)

For the patients underwent ETOCD surgery, compared to the pre-operative peripapillary thickness of peripapillary retinal nerve fiber layer (RNFL) $(103.70 \pm 17.93)$, the post-operative thickness of RNFL significantly decreased 3 months after ETOCD $(82.07 \pm 26.51 ; P<0.001$; Fig. 3a, Table 4) in ITON patients. Meanwhile, the post-operative macular ganglion cell complex (GCC; 68.69 \pm 29.35 ) was significantly thinner than the pre-operative GCC $(86.82 \pm 21.35 ; P<0.001 ;$ Fig. $3 b$, Table 4$)$. Besides, the post-operative vessel density parameters of optic nerve head area decreased significantly 3 months after ETOCD compared with pre-operation, including whole image, inside disk, and radial peripapillary capillary vessel density (all $P<0.001$; Fig. 3c, Table 4 ). Similar to the decreasing tendencies of the vessel density in optic nerve head area, the difference of macular vessel density whole image (m-wiVD) and in each grid session was statistically significant $(P<0.05$; Fig. 3d, Table 4$)$, expect foveal avascular zone (FAZ, $P=0.269$; Table 4 ).

Meanwhile, for the patients treated with conservative therapy, above OCT-A parameters were decreased 


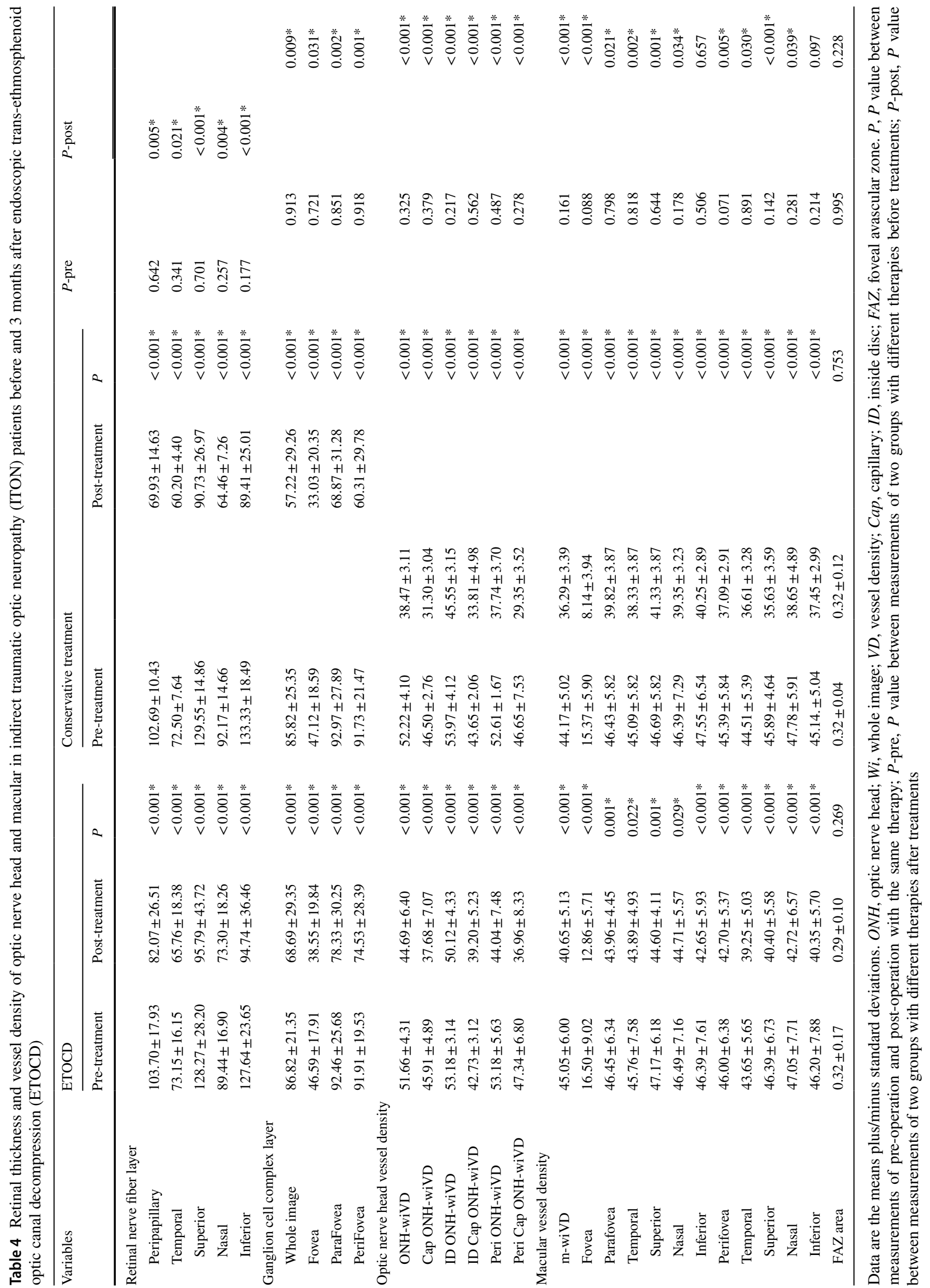



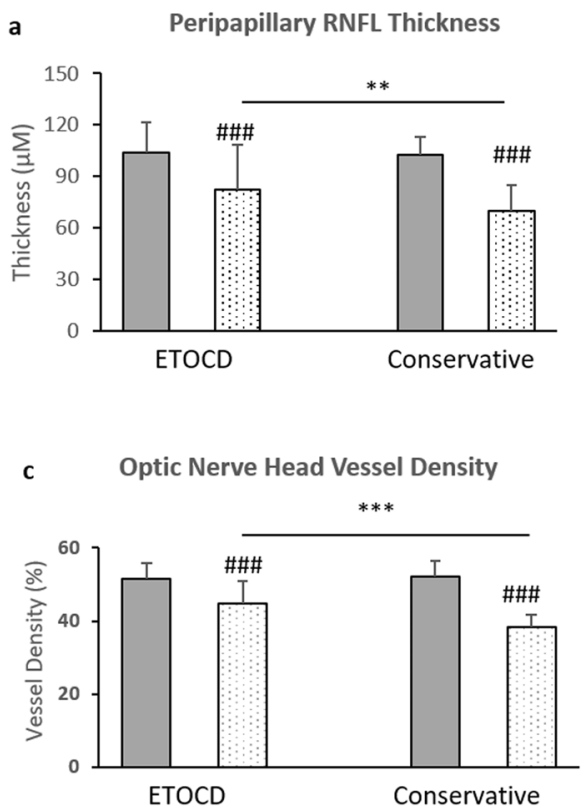

|Pre-treatment

Fig. 3 The thickness and vessel density in optic nerve head area and macular between indirect traumatic optic neuropathy (ITON) patients before and 3 months after endoscopic trans-ethmosphenoid optic canal decompression (ETOCD) and the conservative treatment. Comparison of a the thickness of peripapillary retinal nerve fiber layer (RNFL), $\mathbf{b}$ the thickness of macular ganglion cell complex (GCC), $\mathbf{c}$

significantly 3 months after the treatment compared with pre-treatment (all $P<0.001$; Fig. 3, Table 4), expect FAZ $(P=0.753$; Table 4). Notably, while compared between the two treatment groups after two different therapies, most parameters were found significantly greater in ETOCDtreated group ((peripapillary RNFL thickness: $82.07 \pm 26.51$ vs $69.93 \pm 14.63$; macular GCC thickness: $68.69 \pm 29.35$ vs $57.22 \pm 29.26$; radial peripapillary capillary whole image vessel density: $44.69 \pm 6.40$ vs $38.47 \pm 3.11$; macular whole image vessel density: $40.65 \pm 5.13$ vs $36.29 \pm 3.39$; all $P<0.01$; Fig. 3a-d).

Again, to explore whether the change of vessel density was related to surgical effectiveness, the ITON patients were divided into two groups based on improved VA or not after surgery. Before surgery, the retina thickness and the vessel density of optic nerve head area and macular were not significantly different between the two groups (all $P>0.05)$. Notably, while compared between the two groups after surgery, most parameters were found significantly greater in VA-improved group (peripapillary RNFL thickness: $85.99 \pm 29.14$ vs $72.93 \pm 15.96$ [Fig. $4 \mathrm{a}-\mathrm{c}$ ]; macular GCC thickness: $72.26 \pm 27.59$ vs $57.31 \pm 25.47$ [Fig. 4d-f]; radial peripapillary capillary whole image vessel density: $46.06 \pm 6.95$ vs $41.48 \pm 3.04$ [Fig. $4 \mathrm{~g}-\mathrm{i}$ ]; macular whole image vessel density: $41.34 \pm 5.31$ vs $38.27 \pm 3.15$
Macular GCCL Thickness

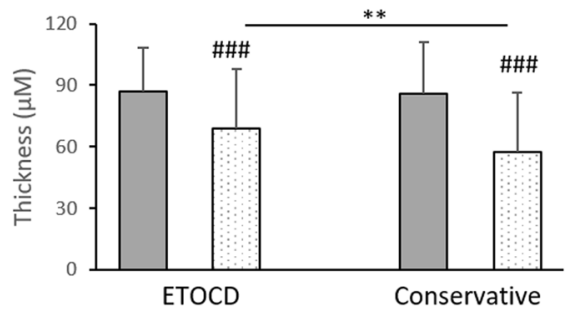

d

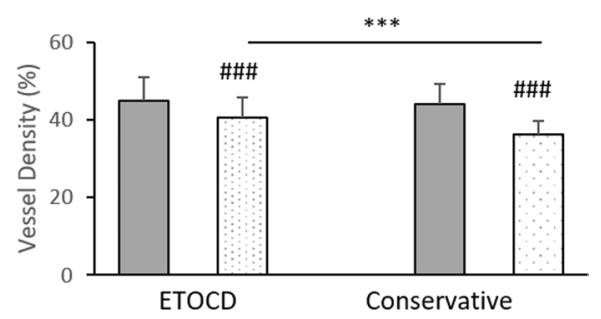

| Post-treatment

the retinal vessel density of optic nerve head area, and $\mathbf{d}$ the retinal vessel density of macular before (gray) and after (dots) treatments, and between groups treated with ETOCD (left) and the conservative treatment (right). ${ }^{\# \# \#} P<0.001$ between values of pre-treatment and 3 months post-treatment. $* * P<0.01$; $* * * P<0.001$ between values of post-ETOCD and post-conservative therapy

[Fig. 4j-1]; all $P<0.01)$. These results showed that VA improvement after surgery related with both retinal thickness and vessel density, which indicated effective surgery, may mitigate the retinal atrophy after severe ITON.

\section{Discussion}

In the present study, we investigated the retinal vasculature alteration in indirect traumatic optic neuropathy (ITON) patients, and found vision recovery after the effective therapy for ITON was associated with the increased oxygen saturation of retinal vessels and the better availability of oxygen in the retina. Moreover, ITON patients who got vision recovery after the effective therapy also presented with thicker retinas and better vessel density, which indicated that effective therapy contributed to mitigate the retinal atrophy of severe ITON. To our best knowledge, this is the first study using oxygen saturation of retinal blood vessels $\left(\mathrm{SO}_{2}\right)$ measurement and optical coherence tomography angiography (OCT-A) to investigate the change of retinal vasculature in ITON individuals.

As an effective and safe therapy for ITON, ETOCD has been widely used as one of the most important treatments of ITON for decades [7-12]. However, the associated 


\section{Improved VA}
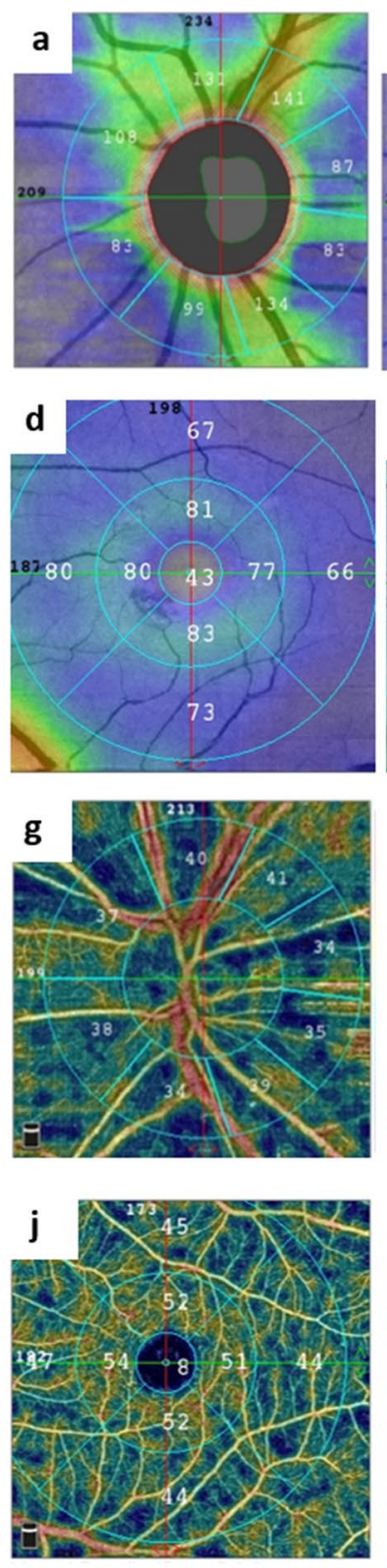

No improved VA
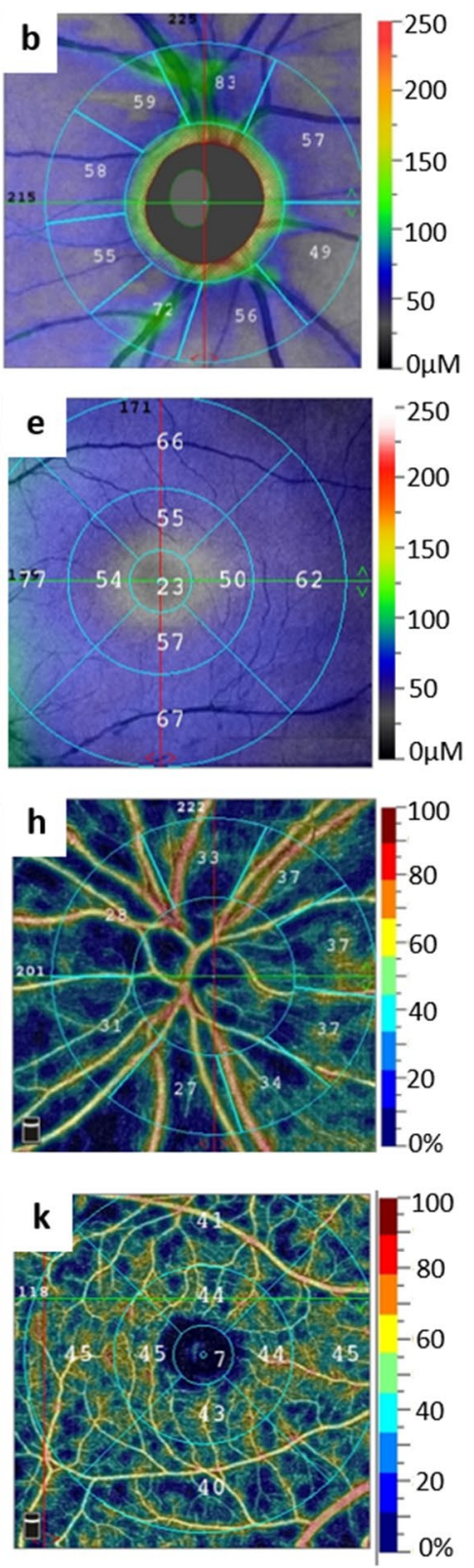

c
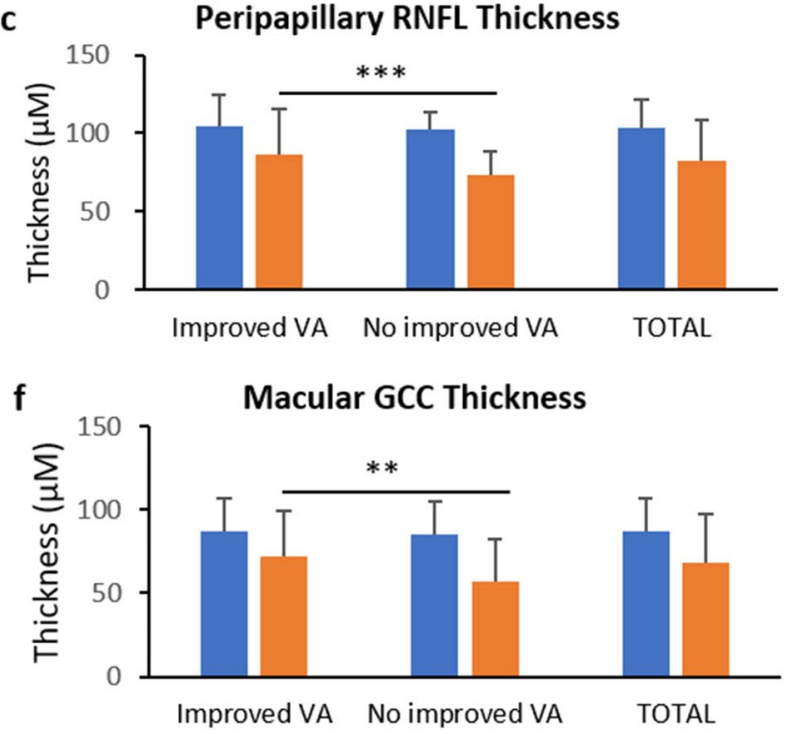

i

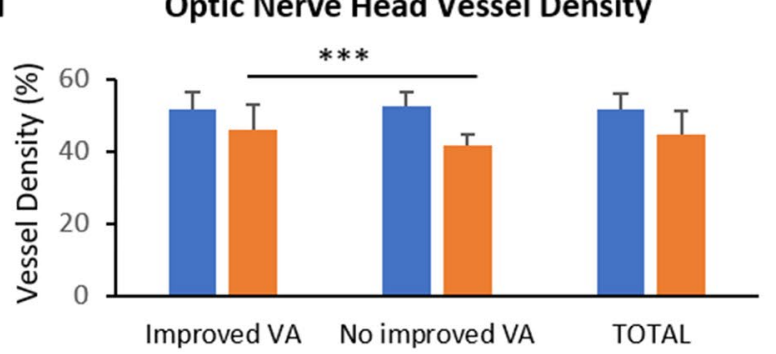

I

Macular Vessel Density

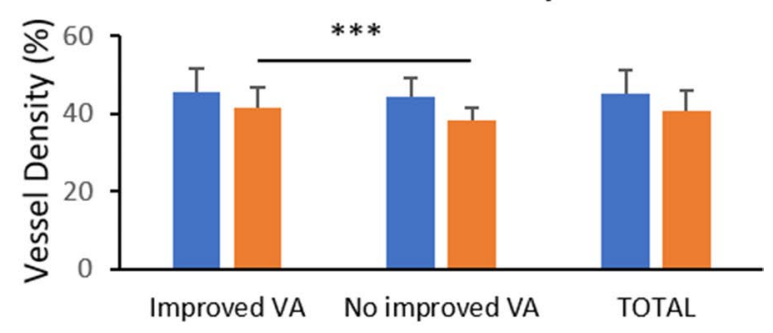

- Pre-operation Post-operation
Fig. 4 The retinal thickness and vessel density in optic nerve head area and macular between indirect traumatic optic neuropathy (ITON) patients with and without VA improvements after endoscopic transethmosphenoid optic canal decompression (ETOCD). (a-b) The typical image of the thickness of peripapillary retinal nerve fiber layer (RNFL) in patients with (a) and without (b) post-operative improved VA after ETOCD. (c) Comparison of peripapillary RNFL before (blue) and after (orange) ETOCD surgery, and between groups of patients with (left) and without (middle) VA improvements. (d-e) The typical image of the thickness of macular ganglion cell complex (GCC) in patients with (d) and without (e) post-operative improved VA after ETOCD. (f) Comparison of macular (GCC) before (blue) and after (orange) ETOCD surgery, and between groups of patients with (left) and without (middle) VA improvements. (g-h) The typical image of retinal vessel density in optic nerve head area of patients with (g) and without (h) post-operative improved VA after ETOCD. (i) Comparison of retinal vessel density in optic nerve head area before (blue) and after (orange) ETOCD surgery, and between groups of patients with (left) and without (middle) VA improvements. (j-k) The typical image of retinal vessel density in macular of patients with (j) and without (k) post-operative improved VA after ETOCD. (l) Comparison of retinal vessel density in macular before (blue) and after (orange) ETOCD surgery, and between groups of patients with (left) and without (middle) VA improvements. $* * P<0.01$; $* * * P<0.001$ between the post-operative parameter for two groups of patients with and without VA improvements 
pathophysiological change and the underlying mechanism of ETOCD were not clear. It has been hypothesized that swelling of the optic nerve within the confined cavity of the optic canal compromises blood supply to the nerve within the canal, which exacerbates tissue ischemia and causes further damage to the injured optic nerve. The damage to the optic nerve due to intraneural edema alters microvasculature and interruption of direct axoplasmic transport. Also, vascular insufficiency resulted from the altered microvasculature might be an important pathogenic mechanism in other optic neuropathies [20, 23, 24], which implies that the abnormal hemodynamic state participates in the course of ITON, and the decompression surgery is effective by improving the retinal vasculature alteration. The spectrophotometric retinal oximetry and the optical coherence tomography angiography (OCT-A) are both noninvasive diagnostic tools to evaluate hemodynamic changes in eye diseases.

In this study, we assessed and analyzed the visual acuity (VA), visual evoked potential (VEP), oxygen saturation of retinal blood vessels, and the vessel density of optic nerve head and macular of 77 ITON patients who received ETOCD surgery and 18 ITON patients who underwent conservative therapies. Our results provided encouraging outcomes of ETOCD surgery, and suggested that it was an optimal choice for effective and safe therapy for ITON patients. Vision improvement is evidenced not only at clinical manifestation levels with multiple measurements of visual acuity but also at functional and pathophysiological and levels as evaluated by VEP, retinal oxygen saturation, and retina vessel density after ETOCD.

In the present study, $76.0 \%$ of patients have vision improved after ETOCD, and $33.3 \%$ of patients have vision improved after the conservative therapy, concordant with the literatures [25-27]. VEP was an important parameter of visual function, especially for the function of the optic nerve. In this study, it was found that VEP values were significantly improved after surgery in ITON patients. Among these main parameters of VEP, the latent period of $\mathrm{N} 2$ and $\mathrm{P} 1$ and the amplitude of $\mathrm{P} 2$ were sensitive to vision recovery in ITON patients.

Furthermore, the oxygen saturation of retinal arteries $\left(\mathrm{SaO}_{2}\right)$ and veins $\left(\mathrm{SvO}_{2}\right)$, as well as the differences between the arteries and veins (AVD), all increased in ITON patients after surgery. The oxygen saturation levels have been shown to associate with multiple ophthalmic conditions. For instance, $\mathrm{SaO}_{2}$ was significantly lower in retinitis pigmentosa patients [21]. In patients with an ischemic branch due to retinal vein occlusion, the occluded arteriole oxygen saturation increased compared to the saturation levels in vessels from the same quadrant in the contralateral eyes [28]. Zheng et al. also found decreased retinal arteriole saturation, a decreased difference in arteriovenous saturation, and a narrowing of retinal vessel diameter in highly myopic eyes [29]. In our study, we found that $\mathrm{SaO}_{2}$ and $\mathrm{AVD}$, representing the use of oxygen by cells, were significantly higher in patients who treated with ETOCD, especially in the patients with VA improvement. Our findings were supportive that the increased oxygen saturation of retinal vessels and the better availability of oxygen in the retina may underlie the vasculature mechanism of VA improvement in ITON patients.

With the emergence of OCT-A, the inspection of retinal microvasculature updated our understanding to the pathogenesis and mechanism of several critical eye diseases, such as glaucoma, ischemic optic neuropathy, and congenital ophthalmopathy [30]. The OCT-A provides measurement of vessel density, which is evidential to the alteration of blood supply following traumatic impact to the optic nerve. To date, the research about the vascular changes in optic neuropathies with OCT-A has been scarce. Chan et al. [6] found that significant decrease in blood supply and oxygenation to the retina was associated with choroidal thinning in chronic ITON patients. Lee et al. [31] reported that in early ITON, a significant thinning of macular ganglion cell complex (GCC) was observed, which implied that GCC loss may participate in the development of ITON. In our study, we found the ETOCD surgery, especially the VA improvement after surgery, was associated with thicker retinas and better vessel density, which indicated that effective decompression surgery may mitigate the progress of retinal atrophy in severe ITON patients.

Our findings suggest that retinal hemodynamic changes, including the increased oxygen saturation of retinal vessels and the better availability of oxygen, were associated with vision recovery after effective treatment of ITON patients. Moreover, the effective treatment of ITON mitigates the progress of retinal atrophy of severe ITON.

Authors' contributions Conceptualization: Rong Lu, Yang Gao; methodology: Yang Gao, Jinmiao Li; formal analysis and investigation: Yang Gao, Jinmiao Li, Cong Nie, Xi Lv; writing (original draft preparation): Yang Gao; writing (review and editing): Rong Lu, Huan Ma; funding acquisition: Rong Lu; resources: Xiaofeng Lin, Guangwei Luo; supervision: Rong Lu, Jianbo Shi.

Funding The work is financially supported by the High-level Hospital Construction Project (303010406) and Natural Science Foundation of Guangdong Province, China (General Program 2019A1515010361). The funding organization had no role in the design or conduct of this research.

Data availability All data and materials support our published claims and comply with field standards.

Code availability All software application or custom code support our published claims and comply with field standards. 


\section{Declarations}

Ethics approval Ethical approval was obtained before treatments and the procedures adhered to the tenets of the 1964 Declaration of Helsinki. Protocols were approved by the Institutional Ethics Committee (2019KYPJ155, Medical Ethics Committee, Zhongshan Ophthalmic Center, Guangzhou, Guangdong, China).

Consent to participate Informed consent was obtained from all individual participants included in the study.

Consent for publication Additional informed consent was obtained from all individual participants for whom identifying information is included in this article.

Conflicts of interest The authors declare no competing interests that are relevant to the content of this article.

Open Access This article is licensed under a Creative Commons Attribution 4.0 International License, which permits use, sharing, adaptation, distribution and reproduction in any medium or format, as long as you give appropriate credit to the original author(s) and the source, provide a link to the Creative Commons licence, and indicate if changes were made. The images or other third party material in this article are included in the article's Creative Commons licence, unless indicated otherwise in a credit line to the material. If material is not included in the article's Creative Commons licence and your intended use is not permitted by statutory regulation or exceeds the permitted use, you will need to obtain permission directly from the copyright holder. To view a copy of this licence, visit http://creativecommons.org/licenses/by/4.0/.

\section{References}

1. al-Qurainy IA, Stassen LF, Dutton GN et al (1991) The characteristics of midfacial fractures and the association with ocular injury: a prospective study. Br J Oral Maxillofac Surg 29:291-301

2. Anderson RL, Panje WR, Gross CE (1982) Optic nerve blindness following blunt forehead trauma. Ophthalmology 89:445-455

3. Jamal BT, Pfahler SM, Lane KA et al (2009) Ophthalmic injuries in patients with zygomaticomaxillary complex fractures requiring surgical repair. J Oral Maxillofac Surg 67:986-989

4. Steinsapir KD, Goldberg RA (2011) Traumatic optic neuropathy: an evolving understanding. Am J Ophthalmol 151(928-933):e922

5. Lee AG (2000) Traumatic optic neuropathy. Ophthalmology 107:814

6. Tong J, Kedar S, Ghate D, Gu L (2019) Indirect traumatic optic neuropathy induced by primary blast: a fluid-structure interaction study. J Biomech Eng 10(1115/1):4043668

7. Yang WG, Chen CT, Tsay PK et al (2004) Outcome for traumatic optic neuropathy-surgical versus nonsurgical treatment. Ann Plast Surg $52: 36-42$

8. Soldevila L, Cano-Parra J, Ruiz A et al (2013) Traumatic optic neuropathy: to treat or not to treat? Report of two cases. Arch Soc Esp Oftalmol 88:116-119

9. Wang AG (2015) How to manage traumatic optic neuropathy? Taiwan J Ophthalmol 5:1-2

10. Ma YJ, Yu B, Tu YH et al (2018) Prognostic factors of trans-ethmosphenoid optic canal decompression for indirect traumatic optic neuropathy. Int J Ophthalmol 11:1222-1226

11. Yu B, Ma Y, Tu Y, Wu W (2016) The outcome of endoscopic transethmosphenoid optic canal decompression for indirect traumatic optic neuropathy with no-light-perception. J Ophthalmol 2016:6492858

12. Lai IL, Liao HT (2018) Risk factor analysis for the outcomes of indirect traumatic optic neuropathy with no light perception at initial visual acuity testing. World Neurosurg 115:e620-e628
13. Hardarson SH, Harris A, Karlsson RA et al (2006) Automatic retinal oximetry. Invest Ophthalmol Vis Sci 47:5011-5016

14. Shoji T, Zangwill LM, Akagi T et al (2017) Progressive macula vessel density loss in primary open-angle glaucoma: a longitudinal study. Am J Ophthalmol 182:107-117

15. Triolo G, Rabiolo A, Shemonski ND et al (2017) Optical coherence tomography angiography macular and peripapillary vessel perfusion density in healthy subjects, glaucoma suspects, and glaucoma patients. Invest Ophthalmol Vis Sci 58:5713-5722

16. Takusagawa HL, Liu L, Ma KN et al (2017) Projection-resolved optical coherence tomography angiography of macular retinal circulation in glaucoma. Ophthalmology 124:1589-1599

17. de Carlo TE, Bonini Filho MA, Chin AT et al (2015) Spectraldomain optical coherence tomography angiography of choroidal neovascularization. Ophthalmology 122:1228-1238

18. Salz DA, de Carlo TE, Adhi M et al (2016) Select features of diabetic retinopathy on swept-source optical coherence tomographic angiography compared with fluorescein angiography and normal eyes. JAMA Ophthalmol 134:644-650

19. Zhang S, Wu C, Liu L et al (2017) Optical coherence tomography angiography of the peripapillary retina in primary angle-closure glaucoma. Am J Ophthalmol 182:194-200

20. Ling JW, Yin X, Lu QY et al (2017) Optical coherence tomography angiography of optic disc perfusion in non-arteritic anterior ischemic optic neuropathy. Int J Ophthalmol 10:1402-1406

21. Zong Y, Lin L, Yi C et al (2016) Retinal vessel oxygen saturation and vessel diameter in retinitis pigmentosa at various ages. Graefes Arch Clin Exp Ophthalmol 254:243-252

22. Li C, Feng X, Wen X et al (2017) Macular retinal vessel oxygen saturation elevation in chinese central serous chorioretinopathy. J Ophthalmol 2017:5972418

23. Augstburger E, Zeboulon P, Keilani C et al (2018) Retinal and choroidal microvasculature in nonarteritic anterior ischemic optic neuropathy: an optical coherence tomography angiography study. Invest Ophthalmol Vis Sci 59:870-877

24. Zhang T, Xiao W, Ye H et al (2019) Peripapillary and macular vessel density in dysthyroid optic neuropathy: an optical coherence tomography angiography study. Invest Ophthalmol Vis Sci 60:1863-1869

25. Yu B, Chen Y, Ma Y et al (2018) Outcome of endoscopic transethmosphenoid optic canal decompression for indirect traumatic optic neuropathy in children. BMC Ophthalmol 18:152

26. Yan W, Lin J, Hu W et al (2020) Combination analysis on the impact of the initial vision and surgical time for the prognosis of indirect traumatic optic neuropathy after endoscopic transnasal optic canal decompression. Neurosurg Rev. https://doi.org/10.1007/ s10143-020-01273-8

27. Huang J, Chen X, Wang Z et al (2020) Selection and prognosis of optic canal decompression for traumatic optic neuropathy. World Neurosurg 138:e564-e578

28. Hardarson SH, Stefansson E (2012) Oxygen saturation in branch retinal vein occlusion. Acta Ophthalmol 90:466-470

29. Zheng Q, Zong Y, Li L et al (2015) Retinal vessel oxygen saturation and vessel diameter in high myopia. Ophthalmic Physiol Opt 35:562-569

30. Cennamo G, Rossi C, Ruggiero P et al (2017) Study of the radial peripapillary capillary network in congenital optic disc anomalies with optical coherence tomography angiography. Am J Ophthalmol 176:1-8

31. Lee JY, Cho K, Park KA, Oh SY (2016) Analysis of retinal layer thicknesses and their clinical correlation in patients with traumatic optic neuropathy. PLoS ONE 11:e0157388

Publisher's note Springer Nature remains neutral with regard to jurisdictional claims in published maps and institutional affiliations. 


\section{Authors and Affiliations}

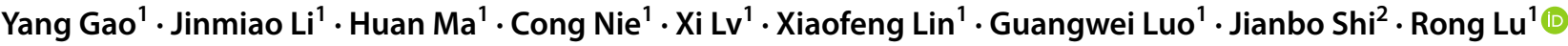

1 State Key Laboratory of Ophthalmology, Zhongshan Ophthalmic Center, Sun Yat-Sen University, 54 Xianlie S. Road, Guangzhou 510060, China
2 Otorhinolaryngology Hospital, The First Affiliated Hospital of Sun Yat-Sen University, Guangzhou 510080, China 